\title{
ROBUST ADAPTIVE TRACKING FOR MARKOVIAN JUMP NONLINEAR SYSTEMS WITH UNKNOWN NONLINEARITIES
}

\author{
JIN ZHU, HONG-SHENG XI, HAI-BO JI, AND BING WANG
}

Received 12 December 2005; Accepted 27 February 2006

Robust adaptive tracking problems for a class of Markovian jump parametric-strict-feedback systems with both parametric uncertainty and unknown nonlinearity are investigated. The unknown nonlinearities considered herein lie within some "bounding functions," which are assumed to be partially known. By using a stochastic Lyapunov method and backstepping techniques, a parameter adaptive law and a control law were obtained, which guarantee that the tracking error could be within a small neighborhood around the origin in the sense of the fourth moment. Moreover, all signals of the closedloop system could be globally uniformly ultimately bounded.

Copyright (C) 2006 Jin Zhu et al. This is an open access article distributed under the Creative Commons Attribution License, which permits unrestricted use, distribution, and reproduction in any medium, provided the original work is properly cited.

\section{Introduction}

The passed decades have witnessed substantial research activities in the development of Markovian jump systems, and much effort is directed towards jump linear systems [6]. With many linear problems (Kalman filtering [4, 10] and LQG [2, 3], etc.) solved, more attention is focused on the study of Markovian jump nonlinear systems. Some results can be found in the works of Aliyu and Boukas [1] and Sathananthan and Keel [9]. And, moreover, Markovian jump nonlinear systems disturbed by Wiener noises (or Brown motion) are becoming the subject of numerous studies in recent years. For this class of jump systems, Mao [5] presents the sufficient condition to ensure existence and uniqueness of the solution; Yuan and Mao $[11,12]$ introduce the notions of stochastic stability. However, at the knowledge of the authors, the practical control design for Markovian jump nonlinear systems has received very little attention in literature.

In this paper, we are interested in the robust adaptive tracking problem for a class of Markovian jump parametric-strict-feedback systems with unknown nonlinearity. The unknown nonlinearity is assumed to satisfy some growth conditions [8]. And the martingale process caused by Markovian jump could be converted to Wiener noises. With the

Hindawi Publishing Corporation

Discrete Dynamics in Nature and Society

Volume 2006, Article ID 92932, Pages 1-18

DOI 10.1155/DDNS/2006/92932 
control law and the parameter adaptive law designed, the tracking error could be within a small neighborhood around the origin in the sense of the fourth moment. And all signals of the closed-loop system are globally uniformly ultimately bounded.

The rest of this paper is organized as follows. Section 2 briefly introduces some mathematic notions and the Markovian jump nonlinear system model. The robust adaptive controller for the system is then proposed in Section 3. In Section 4, an example is shown to illustrate the validity of the design. Finally, conclusions are drawn in Section 5.

\section{Problem and preliminaries}

2.1. Notation. Throughout the paper, unless otherwise specified, we denote by $(\Omega, \mathscr{F}$, $\left.\left\{\mathscr{F}_{t}\right\}_{t \geq 0}, P\right)$ a complete probability space with a filtration $\left\{\mathscr{F}_{t}\right\}_{t \geq 0}$ satisfying the usual conditions (i.e., it is right continuous and $\mathscr{F}_{0}$ contains all $p$-null sets). Let $|\cdot|_{p}$ stand for the $p$ th Euclidean norm for vectors. The superscript $T$ will denote transpose and we refer to $\operatorname{Tr}(\cdot)$ as the trace for matrix. In addition, we use $L^{2}(P)$ to denote the space of Lebesgue square integrable vector.

Let $r(t), t \geq 0$, be a right-continuous Markov chain on the probability space taking values in finite state space $S=\{1,2, \ldots, N\}$, and we introduce $\Phi_{t}=\left[\Phi_{t 1}, \Phi_{t 2}, \ldots, \Phi_{t N}\right]^{T}$, the indicator process for the regime (or mode) $r(t)$, as

$$
\Phi_{t j}= \begin{cases}1, & r(t)=j, \\ 0, & r(t) \neq j, \quad j \in S .\end{cases}
$$

And $\Phi_{t}$ satisfies the following equation:

$$
\Phi_{t}=\Phi_{0}+\Pi \int_{0}^{t} \Phi_{s} d s+M_{t}
$$

with $M_{t}=\left[M_{t 1}, M_{t 2}, \ldots, M_{t N}\right]^{T}$ an $\mathscr{F}_{t}$-martingale satisfying $M_{t} \in L^{2}(P)$ and $\Pi=\left[\pi_{k j}\right]$, the chain generator, an $N \times N$ matrix. The entries $\pi_{k j}, k, j=1,2, \ldots, N$, are interpreted as transition rates such that

$$
P(r(t+d t)=j \mid r(t)=k)= \begin{cases}\pi_{k j} d t+o(d t) & \text { if } k \neq j, \\ 1+\pi_{k j} d t+o(d t) & \text { if } k=j\end{cases}
$$

where $d t>0$. Here $\pi_{k j}>0(k \neq j)$ is the transition rate from $k$ to $j$. Notice that the total probability axiom imposes $\pi_{k k}$ negative and

$$
\sum_{j=1}^{N} \pi_{k j}=0, \quad \forall k \in S
$$


Consider a stochastic differential equation with Markovian switching of the form

$$
d x=f(x, t, r(t)) d t+g(x, t, r(t)) d w
$$

on $t \geq 0$ with initial data $x(0)=x_{0} \in \mathbb{R}^{n}$ and $r(0)=k_{0} \in S$, where $f: \mathbb{R}^{n} \times \mathbb{R}_{+} \times S \rightarrow \mathbb{R}^{n}$ and $g: \mathbb{R}^{n} \times \mathbb{R}_{+} \times S \rightarrow \mathbb{R}^{n \times m} . \omega(t)=\left(\omega_{t 1}, \omega_{t 2}, \ldots, \omega_{t m}\right)^{T}$ is an independent $m$-dimensional standard Wiener noise defined on the probability space. Furthermore, we assume that the Wiener noise $\omega(t)$ is independent of the Markov chain $r(t)$. For the existence and uniqueness of the solution, we will impose a hypothesis (see [5]).

(H) Both $f$ and $g$ satisfy the local Lipschitz condition and the linear growth condition. That is, for each $h=1,2, \ldots$, there is an $L_{h}>0$ such that

$$
|f(x, t, k)-f(y, t, k)| \vee|g(x, t, k)-g(y, t, k)| \leq L_{h}|x-y|
$$

for all $(t, k) \in \mathbb{R}_{+} \times S$ and those $x, y \in \mathbb{R}^{n}$ with $|x| \vee|y| \leq h$. Moreover, there is an $v>0$ such that

$$
|f(x, t, k)| \vee|g(x, t, k)| \leq v(1+|x|)
$$

for all $(x, t, k) \in \mathbb{R}^{n} \times \mathbb{R}_{+} \times S$.

In general, the hypothesis $(\mathrm{H})$ will guarantee a unique local solution to (2.5).

Let $C^{2,1}\left(\mathbb{R}^{n} \times \mathbb{R}_{+} \times S\right)$ denote the family of all functions $F(x, t, k)$ on $\mathbb{R}^{n} \times \mathbb{R}_{+} \times S$ which are continuously twice differentiable in $x$ and once in $t$. Furthermore, we will give the stochastic differentiable equation of $F(x, t, k)$ :

Fix any $\left(x_{0}, t_{0}, k\right) \in \mathbb{R}^{n} \times \mathbb{R}_{+} \times S$ and suppose $x(\mathrm{t})$ is the unique solution to (2.5). By the generalized Ito formula, we have

$$
\begin{aligned}
F(x, t, r(t))= & F\left(x_{0}, t_{0}, k\right)+\int_{t_{0}}^{t} \frac{\partial F(x, s, r(s))}{\partial s} d s+\int_{t_{0}}^{t} \frac{\partial F(x, s, r(s))}{\partial x} f(x, s, r(s)) d s \\
& +\int_{t_{0}}^{t} \frac{1}{2} \operatorname{Tr}\left[g^{T}(x, s, r(s)) \frac{\partial^{2} F(x, s, r(s))}{\partial x^{2}} g(x, s, r(s))\right] d s \\
& +\int_{t_{0}}^{t} \frac{\partial F(x, s, r(s))}{\partial x} g(x, s, r(s)) d \omega \\
& +\int_{t_{0}}^{t} \sum_{j=1}^{N}[F(x, s, j)-F(x, s, k)] d \Phi_{s j} .
\end{aligned}
$$

According to (2.2), the differential equation of the indicator $\Phi_{t}$ is as follows:

$$
d \Phi_{t}=\Pi \Phi_{t} d t+d M_{t}
$$


4 Tracking problems of Markovian jump nonlinear systems

Submit (2.9) into (2.8) and notice that

$$
\sum_{j=1}^{N} \pi_{k j} F(x, t, k)=0 . \quad \forall k \in S .
$$

So

$$
\begin{aligned}
F(x, t, r(t))= & F\left(x_{0}, t_{0}, k\right)+\int_{t_{0}}^{t} \frac{\partial F(x, s, r(s))}{\partial s} d s+\int_{t_{0}}^{t} \frac{\partial F(x, s, r(s))}{\partial x} f(x, s, r(s)) d s \\
& +\int_{t_{0}}^{t} \frac{1}{2} \operatorname{Tr}\left[g^{T}(x, s, r(s)) \frac{\partial^{2} F(x, s, r(s))}{\partial x^{2}} g(x, s, r(s))\right] d s \\
& +\int_{t_{0}}^{t} \frac{\partial F(x, s, r(s))}{\partial x} g(x, s, r(s)) d \omega+\int_{t_{0}}^{t} \sum_{j=1}^{N} \pi_{k j} F(x, s, j) d s \\
& +\int_{t_{0}}^{t} \sum_{j=1}^{N}[F(x, s, j)-F(x, s, k)] d M_{s j} .
\end{aligned}
$$

Therefore, the stochastic differentiable equation of $F(x, t, k)$ is given by the following:

$$
\begin{aligned}
d F(x, t, k)= & \frac{\partial F(x, t, k)}{\partial t} d t+\frac{\partial F(x, t, k)}{\partial x} f(x, t, k) d t \\
& +\frac{1}{2} \operatorname{Tr}\left[\Upsilon^{T} g^{T}(x, t, k) \frac{\partial^{2} F(x, t, k)}{\partial x^{2}} g(x, t, k) \Upsilon\right] d t \\
& +\sum_{j=1}^{N} \pi_{k j} F(x, t, j) d t+\frac{\partial F(x, t, k)}{\partial x} g(x, t, k) d \omega \\
& +\sum_{j=1}^{N}[F(x, t, j)-F(x, t, k)] d M_{t j} .
\end{aligned}
$$

We take the expectation in (2.12), so that the the infinitesimal generator produces [5]

$$
\begin{aligned}
\mathscr{L} F(x, t, k)= & \frac{\partial F(x, t, k)}{\partial t}+\frac{\partial F(x, t, k)}{\partial x} f(x, t, k) \\
& +\frac{1}{2} \operatorname{Tr}\left[g^{T}(x, t, k) \frac{\partial^{2} F(x, t, k)}{\partial x^{2}} g(x, t, k)\right]+\sum_{j=1}^{N} \pi_{k j} F(x, t, j) .
\end{aligned}
$$


Lemma 2.1 (Martingale representation [7]). Let $B(t)=\left[B_{1}(t), B_{2}(t), \ldots, B_{N}(t)\right]$ be $N$ dimensional standard Wiener noise. Suppose $M_{t}$ is an $\mathscr{F}_{t}^{N}$-martingale (with respect to $P$ ) and that $M_{t} \in L^{2}(P)$ for all $t \geq 0$. Then there exists a stochastic process $\Psi \in L^{2}\left(\mathscr{F}_{t}, P\right)$, such that

$$
d M_{t}=\Psi \cdot d B(t)
$$

Lemma 2.2 (Young's inequality). For any two vectors $x, y \in \mathbb{R}^{n}$, the following holds:

$$
x^{T} y \leq \frac{\epsilon^{p}}{p}|x|^{p}+\frac{1}{q \epsilon^{q}}|y|^{q},
$$

where $\epsilon>0$ and the constants $p>1, q>1$ satisfy $(p-1)(q-1)=1$.

2.2. Problem description. Consider the following Markovian jump uncertain nonlinear systems with Wiener noises:

$$
\begin{gathered}
d x_{i}=x_{i+1} d t+\varphi_{i}\left(\bar{x}_{i}, t, r(t)\right)^{T} \theta^{*} d t+\Delta_{i}\left(\bar{x}_{i}, t, r(t)\right) d t \\
d x_{n}=u d t+\varphi_{n}(x, t, r(t))^{T} \theta^{*} d t+\Delta_{n}(x, t, r(t)) d t \\
y=x_{1}, \quad i=1,2, \ldots, n-1,
\end{gathered}
$$

where $x=\left(x_{1}, x_{2}, \ldots, x_{n}\right)^{T} \in \mathbb{R}^{n}$ is the state vector, here $\bar{x}_{i} \triangleq\left(x_{1}, x_{2}, \ldots, x_{i}\right)^{T}, u \in \mathbb{R}$ is the input, and $y \in \mathbb{R}$ is the output of the system. $\theta^{*} \in \mathbb{R}^{P}$ is a vector of unknown constant parameters; The Markov chain $r(t)$ is as defined in Section 2.1. $\varphi_{i}\left(\bar{x}_{i}, t, r(t)\right)$ is a vectorvalued smooth function. $\Delta_{i}\left(\bar{x}_{i}, t, r(t)\right)$ is an unknown function which could be due to modelling errors, parametric uncertainty, time variations in the systems, or a combination of these. And it may be different with each regime $r(t) \in S$. It is assumed that the control designer has, at least, partial knowledge of bounds for the function uncertainty $\Delta_{i}\left(\bar{x}_{i}, t, r(t)\right)$. In particular, we assume that

$$
\left|\Delta_{i}\left(\bar{x}_{i}, t, r(t)=k\right)\right| \leq \psi_{i}^{*} p_{i}\left(\bar{x}_{i}, r(t)=k\right) \quad \forall \bar{x}_{i} \in \mathbb{R}^{i}, \forall t \in \mathbb{R}_{+}, \forall k \in S,
$$

where $p_{i}\left(\bar{x}_{i}, r(t)\right) \in C^{1}\left(\mathbb{R}^{i} \times S, \mathbb{R}_{+}\right)$is a known smooth function and $\psi_{i}^{*} \geq 0$ is a constant parameter, which is not necessarily known. Note that $\psi_{i}^{*}$ is not unique, since any $\bar{\psi}_{i}^{*}>\psi_{i}^{*}$ satisfies inequality (2.17). To avoid confusion, we define $\psi_{i}^{*}$ to be the smallest (nonnegative) constant such that (2.17) is satisfied. In this paper, the equilibrium $x=0$ is assumed to be a common one for all regimes, which means $\varphi_{i}(0, t, k)=0$, for all $k \in S$. With $\varphi_{i}\left(\bar{x}_{i}, t, r(t)\right), \Delta_{i}\left(\bar{x}_{i}, t, r(t)\right)$ satisfying hypothesis $(\mathrm{H})$, Markovian jump system (2.19) has a unique solution. 
Our purpose is to design the controller such that the output $y$ could track a given signal $y_{r}(t)$ (which is supposed to be sufficiently smooth), and moreover all the signals of the closed-loop system could be bounded. We denote the tracking error by

$$
e_{i}=x_{i}-y_{r}^{(j-1)}, \quad j=1,2, \ldots, n \text {. }
$$

Here $y_{r}^{(j)}$ means the $j$ th derivative of $y_{r}$, and we have $y_{r}^{(0)}=y_{r}$. Therefore, the tracking error equations are drawn as

$$
\begin{gathered}
d e_{i}=e_{i+1} d t+\varphi_{i}\left(\bar{x}_{i}, t, r(t)\right)^{T} \theta^{*} d t+\Delta_{i}\left(\bar{x}_{i}, t, r(t)\right) d t \\
d e_{n}=\left(u-y_{r}^{(n)}\right) d t+\varphi_{n}(x, t, r(t))^{T} \theta^{*} d t+\Delta_{n}(x, t, r(t)) d t \\
i=1,2, \ldots, n-1 .
\end{gathered}
$$

\section{Control design}

Now we begin to design a robust adaptive controller for system (2.19) where the parameter $\theta^{*}$ and $\psi_{i}^{*}$ are all needed to be estimated. Denote the estimation of $\theta^{*}$ with $\theta$, and the estimation of $\psi_{i}^{*}$ with $\psi_{i}$.

First we employ a coordinate transformation:

$$
z_{i}=e_{i}-\alpha_{i-1}\left(\bar{x}_{i-1}, \theta, \psi_{i}, t, r(t)=k\right)
$$

where $\alpha_{0}=0$, for all $k \in S$, and the new coordinate is $Z=\left(z_{1}, z_{2}, \ldots, z_{n}\right)$. For simplicity, we denote $\alpha_{i-1}\left(\bar{x}_{i-1}, \theta, \psi_{i}, t, k\right), \varphi_{i}\left(\bar{x}_{i}, t, k\right)$, and $\Delta_{i}\left(\bar{x}_{i}, t, k\right)$ by $\alpha_{i-1}(k), \varphi_{i}(k)$, and $\Delta_{i}(k)$.

According to (2.12), (3.1) can be written as

$$
\begin{aligned}
d z_{i}= & {\left[e_{i+1}+\varphi_{i}^{T}(k) \theta^{*}+\Delta_{i}(k)\right] d t-d \alpha_{i-1}(k) } \\
= & \left\{z_{i+1}+\alpha_{i}(k)+\varphi_{i}^{T}(k) \theta^{*}+\Delta_{i}(k)\right\} d t-\frac{\partial \alpha_{i-1}(k)}{\partial t} d t-\frac{\partial \alpha_{i-1}(k)}{\partial \theta} \dot{\theta} d t \\
& -\sum_{j=1}^{i-1} \frac{\partial \alpha_{i-1}(k)}{\partial \psi_{j}} \dot{\psi}_{j} d t-\sum_{j=1}^{i-1} \frac{\partial \alpha_{i-1}(k)}{\partial x_{j}}\left[x_{j+1}+\varphi_{j}^{T}(k) \theta^{*}+\Delta_{j}(k)\right] d t \\
& -\sum_{j=1}^{N} \pi_{k j} \alpha_{i-1}(j) d t+\sum_{j=1}^{N}\left[\alpha_{i-1}(k)-\alpha_{i-1}(j)\right] d M_{j} .
\end{aligned}
$$

By Lemma 2.1, there exist a $\Psi \in L^{2}\left(\mathscr{F}_{t}, P\right)$ and an $N$-dimensional standard Wiener noise $B(t)$, such that $d M_{t}=\Psi d B(t)$, where $E\left\{\Psi \Psi^{T}\right\}=\phi \phi^{T} \leq Q<\infty$ and $Q$ is a positive 
bounded constant. Moreover, we define a vector $\Gamma_{i}(k) \triangleq\left[\alpha_{i-1}(k)-\alpha_{i-1}(1), \alpha_{i-1}(k)-\right.$ $\left.\alpha_{i-1}(2), \ldots, \alpha_{i-1}(k)-\alpha_{i-1}(N)\right]$. Therefore, (3.2) is as follows:

$$
\begin{aligned}
d z_{i}= & {\left[z_{i+1}+\alpha_{i}(k)+\varphi_{i}^{T}(k) \theta^{*}+\Lambda_{i}(k)\right] d t-\frac{\partial \alpha_{i-1}(k)}{\partial t} d t-\frac{\partial \alpha_{i-1}(k)}{\partial \theta} \dot{\theta} d t } \\
& -\sum_{j=1}^{i-1} \frac{\partial \alpha_{i-1}(k)}{\partial \psi_{j}} \dot{\psi}_{j} d t-\sum_{j=1}^{i-1} \frac{\partial \alpha_{i-1}(k)}{\partial x_{j}}\left[x_{j+1}+\varphi_{j}^{T}(k) \theta^{*}\right] d t \\
& -\sum_{j=1}^{N} \pi_{k j} \alpha_{i-1}(j) d t+\Gamma_{i}(k) \Psi d B,
\end{aligned}
$$

where $\Lambda_{i}(k)$ is

$$
\Lambda_{i}(k) \triangleq \Delta_{i}(k)-\sum_{j=1}^{i-1} \frac{\partial \alpha_{i-1}(k)}{\partial x_{j}} \Delta_{j}(k)
$$

and, according to inequality (2.17), it is easily seen that there exists a series of continuous functions $\bar{p}_{i}\left(\bar{x}_{i}, k\right) \in C\left(\mathbb{R}_{i} \times S, \mathbb{R}_{+}\right)$, such that

$$
\left|\Lambda_{i}(k)\right| \leq \psi_{i}^{*} \bar{p}_{i}\left(\bar{x}_{i}, k\right), \quad \forall \bar{x}_{i} \in \mathbb{R}^{i}, \forall t \in \mathbb{R}_{+}, \forall k \in S .
$$

Choose a Lyapunov function of the form

$$
V=\frac{1}{4} \sum_{i=1}^{n} z_{i}^{4}+\frac{1}{2 \gamma} \tilde{\theta}^{T} \tilde{\theta}+\sum_{i=1}^{n} \frac{1}{2 \sigma_{i}} \chi_{i}^{2}
$$

where $\gamma>0, \sigma_{i}>0$ are constants. $\tilde{\theta}=\theta^{*}-\theta$ and $\chi_{i}=\psi_{i}^{M}-\psi_{i}$ are the parameter estimation errors, where $\psi_{i}^{M} \triangleq \max \left\{\psi_{i}^{*}, \psi_{i}^{0}\right\}$, and $\psi_{i}^{0}$ are given positive constants.

We set out to choose the function $\alpha_{i-1}\left(\bar{x}_{i-1}, \theta, \psi_{i}, t, k\right)$ and adaptive functions to make $\mathscr{L V}$ nonpositive. Along the solutions of (3.3), we have

$$
\begin{aligned}
\mathscr{L} V= & \sum_{i=1}^{n} z_{i}^{3}\left\{z_{i+1}+\alpha_{i}(k)+\varphi_{i}^{T}(k) \theta^{*}-\frac{\partial \alpha_{i-1}(k)}{\partial t}-\frac{\partial \alpha_{i-1}(k)}{\partial \theta} \dot{\theta}-\sum_{j=1}^{i-1} \frac{\partial \alpha_{i-1}(k)}{\partial \psi_{j}} \dot{\psi}_{j}\right. \\
& \left.-\sum_{j=1}^{N} \pi_{k j} \alpha_{i-1}(j)-\sum_{j=1}^{i-1} \frac{\partial \alpha_{i-1}(k)}{\partial x_{j}}\left[x_{j+1}+\varphi_{j}^{T}(k) \theta^{*}\right]+\Lambda_{i}(k)\right\} \\
& +\frac{3}{2} \sum_{i=1}^{N} z_{i}^{2} \Gamma_{i}(k) \phi \phi^{T} \Gamma_{i}^{T}(k)-\frac{1}{\gamma} \tilde{\theta}^{T} \dot{\theta}-\sum_{i=1}^{n} \frac{1}{\sigma_{i}} \chi_{i} \dot{\psi}_{i}
\end{aligned}
$$




$$
\begin{aligned}
\leq \sum_{i=1}^{N} z_{i}^{3}\left\{\left(\frac{3}{4} \delta_{i}^{4 / 3}+\frac{1}{4 \delta_{i-1}^{4}}\right) z_{i}+\alpha_{i}(k)-\frac{\partial \alpha_{i-1}(k)}{\partial t}-\frac{\partial \alpha_{i-1}(k)}{\partial \theta} \dot{\theta}-\sum_{j=1}^{i-1} \frac{\partial \alpha_{i-1}(k)}{\partial \psi_{j}} \dot{\psi}_{j}\right. \\
\left.+\tau_{i}^{T}(k) \theta-\sum_{j=1}^{i-1} \frac{\partial \alpha_{i-1}(k)}{\partial x_{j}} x_{j+1}-\sum_{j=1}^{N} \pi_{k j} \alpha_{i-1}(j)+\sum_{i=1}^{n} \mu z_{i}\left[\Gamma_{i}(k) \Gamma_{i}^{T}(k)\right]^{2}\right\} \\
+\sum_{i=1}^{n} \frac{9}{16 \mu} Q^{2}-\tilde{\theta}^{T}\left[\frac{1}{\gamma} \dot{\theta}-\sum_{i=1}^{n} z_{i}^{3} \tau_{i}(k)\right]-\sum_{i=1}^{n}\left[\frac{1}{\sigma_{i}} \chi_{i} \dot{\psi}_{i}-z_{i}^{3} \Lambda_{i}(k)\right]
\end{aligned}
$$

with

$$
\tau_{i}(k)=\varphi_{i}(k)-\sum_{j=1}^{i-1} \frac{\partial \alpha_{i-1}(k)}{\partial x_{j}} \varphi_{j}(k)
$$

In (3.7), the following inequalities are used, which can be reduced from Young's inequalities and norm inequalities with the help of changing the order of summations or exchanging the indices of the summations:

$$
\sum_{i=1}^{n} z_{i}^{3} z_{i+1} \leq \frac{3}{4} \sum_{i=1}^{n-1} \delta^{4 / 3} z_{i}^{4}+\frac{1}{4} \sum_{i=1}^{n-1} \frac{1}{\delta_{i}^{4}} z_{i+1}^{4}=\sum_{i=1}^{n}\left(\frac{3}{4} \delta_{i}^{4 / 3}+\frac{1}{4 \delta_{i-1}^{4}}\right) z_{i}^{4}
$$

where $\delta_{0}=\infty, \delta_{n}=0$, and $\delta_{i}>0, i=1,2, \ldots, n-1$,

$$
\begin{aligned}
\frac{3}{2} \sum_{i=1}^{n} z_{i}^{2} \Gamma_{i}(k) \phi \phi^{T} \Gamma_{i}^{T}(k) & \leq \frac{3}{2} \sum_{i=1}^{n} z_{i}^{2} \Gamma_{i}(k) Q \Gamma_{i}^{T}(k) \\
& \leq \sum_{i=1}^{n} \mu z_{i}^{4}\left[\Gamma_{i}(k) \Gamma_{i}^{T}(k)\right]^{2}+\sum_{i=1}^{n} \frac{9}{16 \mu} Q^{2}
\end{aligned}
$$

where $\lambda>0, \mu>0$ are design parameters.

According to [8], we suggest the following adaptive laws:

$$
\begin{aligned}
& \dot{\theta}=\gamma\left[\sum_{i=1}^{n} z_{i}^{3} \tau_{i}(k)-l\left(\theta-\theta^{0}\right)\right], \\
& \dot{\psi}_{i}=\sigma_{i}\left[z_{i}^{3} \omega_{i}(k)-m_{i}\left(\psi_{i}-\psi_{i}^{0}\right)\right], \\
& \omega_{i}(k)=\bar{p}_{i}(k) \cdot \tanh \left[\frac{z_{i}^{3} \bar{p}_{i}(k)}{\epsilon_{i}}\right] .
\end{aligned}
$$


Jin Zhu et al. 9

Here $l>0, m_{i}>0, \epsilon_{i}>0, \theta^{0} \in \mathbb{R}^{p}$ are given constants. Denote

$$
\beta_{i}(k)=\psi_{i} \cdot \omega_{i}(k)
$$

Substituting (3.11), (3.12), and (3.14) into (3.7), we suggest the virtual control as

$$
\begin{aligned}
\alpha_{i}(k)= & -c_{i} z_{i}-\left(\frac{3}{4} \delta_{i}^{4 / 3}+\frac{1}{4 \delta_{i-1}^{4}}\right) z_{i}+\frac{\partial \alpha_{i-1}(k)}{\partial t}-\mu z_{i}\left[\Gamma_{i}(k) \Gamma_{i}^{T}(k)\right]^{2}+\frac{\partial \alpha_{i-1}(k)}{\partial \theta} \dot{\theta} \\
& +\sum_{j=1}^{i-1} \frac{\partial \alpha_{i-1}(k)}{\partial \psi_{j}} \dot{\psi}_{j}(k)-\tau_{i}^{T}(k) \theta+\sum_{j=1}^{i-1} \frac{\partial \alpha_{i-1}(k)}{\partial x_{j}} x_{j+1}+\sum_{j=1}^{N} \pi_{k j} \alpha_{i-1}(j)-\beta_{i}(k) .
\end{aligned}
$$

However, if adaptive law (3.11) is adopted, $\dot{\theta}$ concerning with $z_{1}, \ldots, z_{n}$ exists in (3.15). Therefore, it is impossible to get $\alpha_{i}(k)$ directly. For this reason, the following transitions are necessary:

$$
\begin{aligned}
\sum_{i=1}^{n} z_{i}^{3} \frac{\partial \alpha_{i-1}(k)}{\partial \theta} \dot{\theta}= & \sum_{i=1}^{n} z_{i}^{3} \frac{\partial \alpha_{i-1}(k)}{\partial \theta} \gamma\left[\sum_{j=1}^{i} z_{j}^{3} \tau_{j}(k)+\sum_{j=i+1}^{n} z_{j}^{3} \tau_{j}(k)-l\left(\theta-\theta^{0}\right)\right] \\
= & \sum_{i=1}^{n} z_{i}^{3} \frac{\partial \alpha_{i-1}(k)}{\partial \theta} \sum_{j=1}^{i} z_{j}^{3} \tau_{j}(k)+\sum_{j=1}^{n} \sum_{i=1}^{j-1} z_{i}^{3} \frac{\partial \alpha_{i-1}(k)}{\partial \theta} \gamma z_{j}^{3} \tau_{j}(k) \\
& -\sum_{i=1}^{n} z_{i}^{3} \frac{\partial \alpha_{i-1}(k)}{\partial \theta} \gamma l\left(\theta-\theta^{0}\right) \\
= & \gamma \sum_{i=1}^{n} z_{i}^{3}\left[\frac{\partial \alpha_{i-1}(k)}{\partial \theta} \sum_{j=1}^{i} z_{j}^{3} \tau_{j}(k)+\left(\sum_{j=1}^{i-1} z_{j}^{3} \frac{\partial \alpha_{j-1}(k)}{\partial \theta}\right) \tau_{i}(k)-l\left(\theta-\theta^{0}\right)\right] .
\end{aligned}
$$

Substituting (3.16) into (3.15), the virtual control design is

$$
\begin{aligned}
\alpha_{i}(k)= & -c_{i} z_{i}-\left(\frac{3}{4} \delta_{i}^{4 / 3}+\frac{1}{4 \delta_{i-1}^{4}}\right) z_{i}+\frac{\partial \alpha_{i-1}(k)}{\partial t}+\gamma \frac{\partial \alpha_{i-1}(k)}{\partial \theta} \sum_{j=1}^{i} z_{j}^{3} \tau_{j}(k) \\
& +\gamma \sum_{j=1}^{i-1} z_{j}^{3} \frac{\partial \alpha_{j-1}(k)}{\partial \theta} \tau_{i}(k)-\gamma l \frac{\partial \alpha_{i-1}(k)}{\partial \theta}\left(\theta-\theta^{0}\right)+\sum_{j=1}^{i-1} \frac{\partial \alpha_{i-1}(k)}{\partial \psi_{j}} \dot{\psi}_{j} \\
& -\tau_{i}^{T}(k) \theta-\mu z_{i}\left[\Gamma_{i}(k) \Gamma_{i}^{T}(k)\right]^{2}+\sum_{j=1}^{i-1} \frac{\partial \alpha_{i-1}(k)}{\partial x_{j}} x_{j+1}+\sum_{j=1}^{N} \pi_{k j} \alpha_{i-1}(j)-\beta_{i}(k),
\end{aligned}
$$


10 Tracking problems of Markovian jump nonlinear systems

where $\alpha_{0}(k)=0, z_{i}=x_{i}-y_{r}^{(i-1)}-\alpha_{i-1}(k), c_{i}>0, i=1,2, \ldots, n$, with the actual control

$$
u(k)=\alpha_{n}(k)+y_{r}^{(n)}
$$

then the infinitesimal generator of $V$ becomes

$$
\begin{aligned}
\mathscr{L} V \leq & -\sum_{i=1}^{n} c_{i} z_{i}^{4}-\sum_{i=1}^{n}\left[z_{i}^{3} \beta_{i}(k)+z_{i}^{3} \chi_{i}{\omega_{i}}_{i}(k)-z_{i}^{3} \Lambda_{i}(k)-\chi_{i} m_{i}\left(\psi_{i}-\psi_{i}^{0}\right)\right] \\
& +l \tilde{\theta}^{T}\left(\theta-\theta^{0}\right)+\frac{9 n}{16 \mu} Q^{2} \\
= & -\sum_{i=1}^{n} c_{i} z_{i}^{4}+l \tilde{\theta}^{T}\left(\theta-\theta^{0}\right)+\frac{9 n}{16 \mu} Q^{2}+\sum_{i=1}^{n}\left[z_{i}^{3} \Lambda_{i}(k)-\left(\psi_{i}+\chi_{i}\right) z_{i}^{3} \omega_{i}(k)\right] \\
& +\sum_{i=1}^{n} m_{i} \chi_{i}\left(\psi_{i}-\psi_{i}^{0}\right) \\
= & -\sum_{i=1}^{n} c_{i} z_{i}^{4}+l \tilde{\theta}^{T}\left(\theta-\theta^{0}\right)+\frac{9 n}{16 \mu} Q^{2}+\sum_{i=1}^{n}\left(z_{i}^{3} \Lambda_{i}(k)-\psi_{i}^{M} z_{i}^{3} \omega_{i}(k)\right) \\
& +\sum_{i=1}^{n} m_{i} \chi_{i}\left(\psi_{i}-\psi_{i}^{0}\right) .
\end{aligned}
$$

Considering (3.5) and (3.13), we get

$$
\begin{aligned}
& z_{i}^{3} \Lambda_{i}(k)-\psi_{i}^{M} z_{i}^{3} \omega_{i}(k) \\
& \quad \leq \psi_{i}^{*}\left|z_{i}^{3} \bar{p}_{i}(k)\right|-\psi_{i}^{M} z_{i}^{3} \omega_{i}(k) \\
& \quad \leq \psi_{i}^{M}\left|z_{i}^{3} \bar{p}_{i}(k)\right|-\psi_{i}^{M} z_{i}^{3} \bar{p}_{i}(k) \tanh \left[\frac{z_{i}^{3} \bar{p}_{i}(k)}{\varepsilon_{i}}\right]
\end{aligned}
$$

according to

$$
0 \leq|\eta|-\eta \tanh \left(\frac{\eta}{\varepsilon}\right) \leq 0.2785 \varepsilon<\frac{1}{2} \varepsilon
$$

such that

$$
z_{i}^{3} \Lambda_{i}(k)-\psi_{i}^{M} z_{i}^{3} \omega_{i}(k) \leq \frac{1}{2} \psi_{i}^{M} \varepsilon_{i} .
$$


Jin Zhu et al. 11

By using the inequalities

$$
\begin{aligned}
\tilde{\theta}^{T}\left(\theta-\theta^{0}\right) & =-\frac{1}{2} l \tilde{\theta}^{T} \tilde{\theta}-\frac{1}{2} l\left(\theta-\theta^{0}\right)^{T}\left(\theta-\theta^{0}\right)+\frac{1}{2} l\left(\theta^{*}-\theta^{0}\right)^{T}\left(\theta^{*}-\theta^{0}\right) \\
& \leq-\frac{1}{2} l \tilde{\theta}^{T} \tilde{\theta}+\frac{1}{2} l\left(\theta^{*}-\theta^{0}\right)^{T}\left(\theta^{*}-\theta^{0}\right) \\
m_{i} \chi_{i}\left(\psi_{i}-\psi_{i}^{0}\right) & =-\frac{1}{2} m_{i} \chi_{i}^{2}-\frac{1}{2} m_{i}\left(\psi_{i}-\psi_{i}^{0}\right)^{2}+\frac{1}{2} m_{i}\left(\psi_{i}^{M}-\psi_{i}^{0}\right)^{2} \\
& \leq-\frac{1}{2} m_{i} \chi_{i}^{2}+\frac{1}{2} m_{i}\left(\psi_{i}^{M}-\psi_{i}^{0}\right)^{2}
\end{aligned}
$$

therefore

$$
\begin{aligned}
\mathscr{L} V \leq & -\sum_{i=1}^{n} c_{i} z_{i}^{4}-\frac{1}{2} l \tilde{\theta}^{T} \tilde{\theta}+\frac{1}{2} l\left(\theta^{*}-\theta^{0}\right)^{T}\left(\theta^{*}-\theta^{0}\right) \\
& +\frac{1}{2} \sum_{i=1}^{n} \varepsilon_{i} \psi_{i}^{M}-\frac{1}{2} m \sum_{i=1}^{n} \chi_{i}^{2}+\frac{1}{2} \sum_{i=1}^{n} m_{i}\left(\psi_{i}^{M}-\psi_{i}^{0}\right)^{2}+\frac{9 n}{16 \mu} Q^{2} \leq-c V+\kappa,
\end{aligned}
$$

where

$$
\begin{gathered}
m=\min \left(m_{i}\right), \\
c=\min \left(4 c_{i}, l \gamma, m \sigma_{i}\right), \\
\kappa=\frac{1}{2} \sum_{i=1}^{n} \varepsilon_{i} \psi_{i}^{M}+\frac{1}{2} \sum_{i=1}^{n} m_{i}\left(\psi_{i}^{M}-\psi_{i}^{0}\right)^{2} \\
+\frac{1}{2} l\left(\theta^{*}-\theta^{0}\right)^{T}\left(\theta^{*}-\theta^{0}\right)+\frac{9 n}{16 \mu} Q^{2} .
\end{gathered}
$$

Theorem 3.1. The equilibrium of the closed-loop Markovian jump system (2.19), (3.1), (3.11), (3.12), (3.18) is globally uniformly ultimately bounded in the fourth moment. Furthermore, for any given $\varepsilon>0$, there is

$$
\lim _{t \rightarrow \infty} E\left(|Z|_{4}^{4}\right)<\varepsilon
$$


12 Tracking problems of Markovian jump nonlinear systems

Proof. According to the conclusion in [12], we have

$$
E V \leq e^{-c t}\left[V\left(x_{0}, t_{0}, r_{0}\right)-\frac{\kappa}{c}\right]+\frac{\kappa}{c}
$$

and there is

$$
V=\frac{1}{4} \sum_{i=1}^{n} z_{i}^{4}+\frac{1}{2 \gamma} \tilde{\theta}^{T} \tilde{\theta}+\sum_{i=1}^{n} \frac{1}{2 \sigma_{i}} \chi_{i}^{2} \geq \frac{1}{4} \sum_{i=1}^{n} z_{i}^{4} .
$$

Taking expectation in the above equation,

$$
E\left(|Z|_{4}^{4}\right) \leq 4 E V \leq 4 e^{-c t}\left[V\left(x_{0}, t_{0}, r_{0}\right)-\frac{\kappa}{c}\right]+\frac{4 \kappa}{c},
$$

which means that $Z=\left(z_{1}, z_{2}, \ldots, z_{n}\right)$ is globally uniformly bounded in the fourth moment, thus $e=\left(e_{1}, e_{2}, \ldots, e_{n}\right)$ is globally uniformly bounded in the fourth moment.

Moreover, there exists $T>0$, if $t \geq T$, there is $4 e^{-c t}\left[V\left(x_{0}, t_{0}, r_{0}\right)-\kappa / c\right] \leq 4 \kappa / c$, and $E|Z|_{4}^{4} \leq 8 \kappa / c$. So, for any given $\varepsilon>0$, appropriate control design parameters $c_{i}, l, m_{i}$ can be chosen to guarantee $8 \kappa / c<\varepsilon$.

Therefore, when $t \geq T$,

$$
E\left|e_{1}\right|_{4}^{4}=E\left|y-y_{r}\right|_{4}^{4} \leq E|Z|_{4}^{4} \leq \frac{8 \kappa}{c}<\varepsilon,
$$

which means all the signals of the closed-loop system are globally uniformly ultimately bounded in the fourth moment, and we achieve regulation of the tracking error to any prescribed accuracy.

\section{Example}

Consider a two-order Markovian jump nonlinear system with the regime transition space $S=\{1,2\}$, and the transition rate matrix is

$$
\Pi=\left[\begin{array}{cc}
-2 & 2 \\
5 & -5
\end{array}\right]
$$

The system is as follows:

$$
\begin{gathered}
d x_{1}=x_{2} d t+\xi_{1}\left(x_{1}, t, r(t)\right) \theta^{*} d t+\Delta\left(x_{1}, t, r(t)\right) d t \\
d x_{2}=u d t+\xi_{2}(x, t, r(t)) \theta^{*} d t, \\
y=x_{1} .
\end{gathered}
$$


Here

$$
\begin{aligned}
& \xi_{1}\left(x_{1}, t, 1\right)=x_{1}^{2}, \quad \xi_{1}\left(x_{1}, t, 2\right)=x_{1}, \\
& \xi_{2}(x, t, 1)=x_{1}, \quad \xi_{2}(x, t, 2)=x_{1} \sin x_{2},
\end{aligned}
$$

where $\theta^{*}$ is an unknown parameter and $\Delta\left(x_{1}, t, r(t)\right)$ is an unknown bounded disturbance. For simulation purposes, we let $\theta^{*}=2, \Delta\left(x_{1}, t, 1\right)=0.6 \sin 2 t, \Delta\left(x_{1}, t, 2\right)=\cos 4 t$.

The control law and the adaptive law are taken as follows (here $\delta_{1}=1$ ).

Case 1. The system regime is 1 :

$$
\begin{gathered}
\alpha_{1}(1)=-\left(c_{1}+\frac{3}{4}\right) z_{1}-\tau_{1}(1) \theta-\beta_{1}(1) \\
\alpha_{2}(1)=-\left(c_{2}+\frac{1}{4}\right) z_{2}-\tau_{2}(1) \theta-x_{1}^{2} \gamma\left[z_{1}^{3} \tau_{1}(1)+z_{2}^{3} \tau_{2}(1)\right]+\gamma l\left(\theta-\theta^{0}\right) x_{1}^{2}-\beta_{2}(1) \\
-\frac{\partial \alpha_{1}(1)}{\partial x_{1}} x_{2}-\omega_{1}(1) \dot{\psi}_{1}+\pi_{11} \alpha_{1}(1)+\pi_{12} \alpha_{1}(2)-\mu z_{2}\left[\alpha_{1}(1)-\alpha_{1}(2)\right]^{4} \\
\dot{\theta}=\gamma\left[z_{1}^{3} \tau_{1}(1)+z_{2}^{3} \tau_{2}(1)-l\left(\theta-\theta^{0}\right)\right] \\
\dot{\psi_{1}}=\sigma_{1}\left[z_{1}^{3} w_{1}(1)-m_{1}\left(\psi_{1}-\psi_{1}^{0}\right)\right] \\
\dot{\psi_{2}}=\sigma_{2}\left[z_{2}^{3} w_{2}(1)-m_{2}\left(\psi_{2}-\psi_{2}^{0}\right)\right]
\end{gathered}
$$

where

$$
\begin{gathered}
z_{1}=x_{1}-y_{r}, \quad z_{2}=x_{2}-\dot{y}_{r}-\alpha_{1}(1), \quad \tau_{1}(1)=x_{1}^{2}, \quad \bar{p}_{1}(1)=1, \\
\omega_{1}(1)=\bar{p}_{1}(1) \tanh \left(\frac{z_{1}^{3} \bar{p}_{1}(1)}{\epsilon_{1}}\right), \quad \beta_{1}(1)=\psi_{1} \omega_{1}(1), \\
\bar{p}_{2}(1)=\left|\frac{\partial \alpha_{1}(1)}{\partial x_{1}}\right|=\left|c_{1}+\frac{3}{4}+2 \theta x_{1}+\frac{3 z_{1}^{2} \psi_{1}}{\varepsilon_{1}}\left[1-\omega_{1}^{2}(1)\right]\right|, \quad \tau_{2}(1)=x_{1}-\frac{\partial \alpha_{1}(1)}{\partial x_{1}} x_{1}^{2}, \\
\omega_{2}(1)=\bar{p}_{2}(1) \tanh \left(\frac{z_{2}^{3} \bar{p}_{2}(1)}{\varepsilon_{2}}\right), \quad \beta_{2}(1)=\psi_{2} \omega_{2}(1) .
\end{gathered}
$$

Case 2. The system regime is 2 :

$$
\begin{gathered}
\alpha_{1}(2)=-\left(c_{1}+\frac{3}{4}\right) z_{1}-\tau_{1}(2) \theta-\beta_{1}(2), \\
\alpha_{2}(2)=-\left(c_{2}+\frac{1}{4}\right) z_{2}-\tau_{2}(2) \theta-x_{1} \gamma\left[z_{1}^{3} \tau_{1}(2)+z_{2}^{3} \tau_{2}(2)\right]
\end{gathered}
$$


14 Tracking problems of Markovian jump nonlinear systems

$$
\begin{aligned}
& +\gamma l\left(\theta-\theta^{0}\right) x_{1}-\beta_{2}(2)-\frac{\partial \alpha_{1}(2)}{\partial x_{1}} x_{2}-\omega_{1}(2) \dot{\psi}_{1} \\
& +\pi_{21} \alpha_{1}(1)+\pi_{22} \alpha_{1}(2)-\mu z_{2}\left[\alpha_{1}(1)-\alpha_{1}(2)\right]^{4} \\
& \dot{\theta}=\gamma\left[z_{1}^{3} \tau_{1}(2)+z_{2}^{3} \tau_{2}(2)-l\left(\theta-\theta^{0}\right)\right] \\
& \dot{\psi}_{1}=\sigma_{1}\left[z_{1}^{3} w_{1}(2)-m_{1}\left(\psi_{1}-\psi_{1}^{0}\right)\right] \\
& \dot{\psi_{2}}=\sigma_{2}\left[z_{2}^{3} w_{2}(2)-m_{2}\left(\psi_{2}-\psi_{2}^{0}\right)\right],
\end{aligned}
$$

where

$$
\begin{gathered}
z_{1}=x_{1}-y_{r}, \quad z_{2}=x_{2}-\dot{y}_{r}-\alpha_{1}(2), \\
\tau_{1}(2)=x_{1}, \quad \bar{p}_{1}(2)=1, \\
\omega_{1}(2)=\bar{p}_{1}(2) \tanh \left(\frac{z_{1}^{3} \bar{p}_{1}(2)}{\varepsilon_{1}}\right), \quad \beta_{1}(2)=\psi_{1} \omega_{1}(2), \\
\bar{p}_{2}(2)=\left|\frac{\partial \alpha_{1}(2)}{\partial x_{1}}\right|=\left|c_{1}+\frac{3}{4}+\theta+\frac{3 z_{1}^{2} \psi_{1}}{\varepsilon_{1}}\left[1-\omega_{1}^{2}(2)\right]\right|, \\
\tau_{2}(2)=x_{1} \sin x_{2}-\frac{\partial \alpha_{1}(2)}{\partial x_{1}} x_{1}, \\
\omega_{2}(2)=\bar{p}_{2}(2) \tanh \left(\frac{z_{2}^{3} \bar{p}_{2}(2)}{\varepsilon_{2}}\right), \quad \beta_{2}(2)=\psi_{2} \omega_{2}(2) .
\end{gathered}
$$

In computation, we take design constants $c_{1}=c_{2}=5, \theta^{0}=1, \psi_{1}^{0}=\psi_{2}^{0}=1, \sigma_{1}=\sigma_{2}=$ $\gamma=1, m_{1}=m_{2}=l=1, \mu=25, \varepsilon_{1}=\varepsilon_{2}=0.4$, choose the initial values to be $x_{1}=0, x_{2}=0$, $\theta(0)=0, \psi_{1}(0)=\psi_{2}(0)=0$.

When the the given signal to be tracked is $y_{r}=1$, Figure 4.1 shows the regime transition in set-point tracking, Figure 4.2 shows the corresponding control in set-point tracking, and Figure 4.3 shows the time responses of the output variable in set-point tracking (dashed line for output $y$, and solid line for given signal).

When the given signal to be tracked is $y_{r}=0.5+0.2 \sin 5 t$, Figure 4.4 shows the regime transition in periodic-signal tracking, Figure 4.5 shows the corresponding control in periodic-signal tracking, and Figure 4.6 shows the time responses of the output variable in periodic signal tracking. (dashed line for output $y$, and solid line for given signal). 


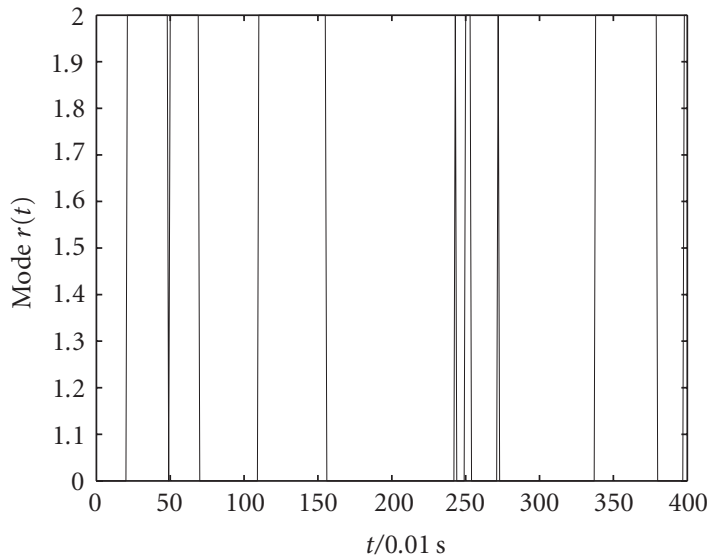

Figure 4.1. Regime transition in set-point tracking.

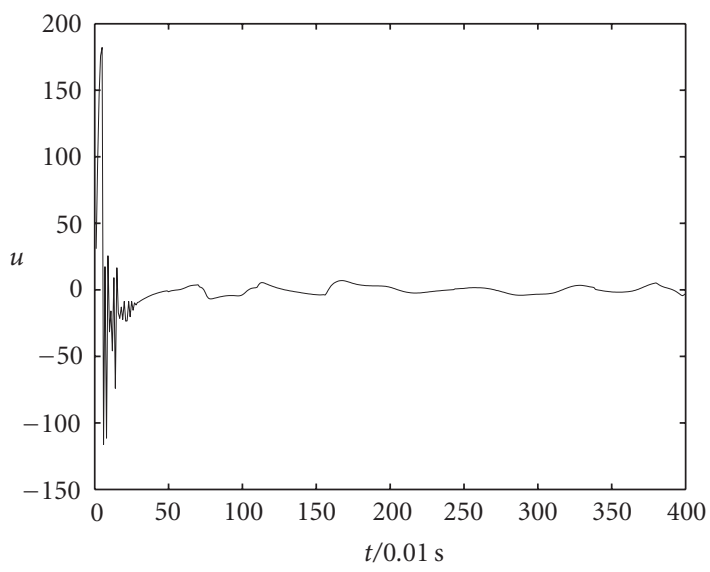

Figure 4.2. Control in set-point tracking.

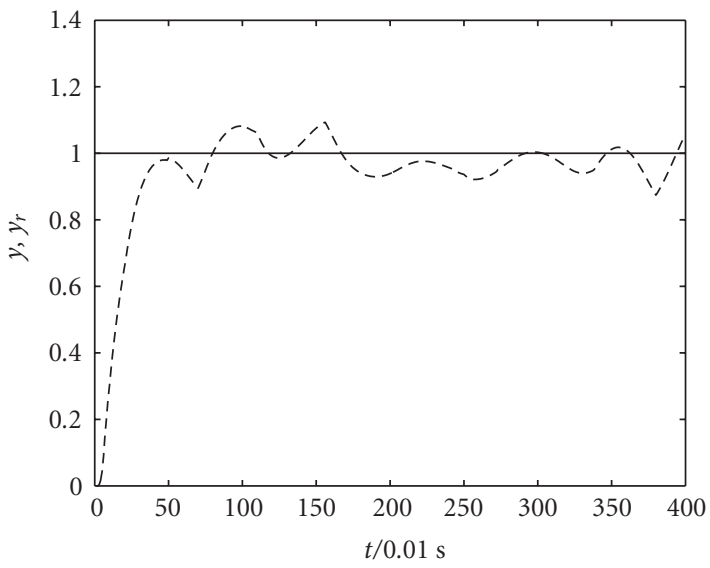

Figure 4.3. Output in set-point tracking. 
16 Tracking problems of Markovian jump nonlinear systems

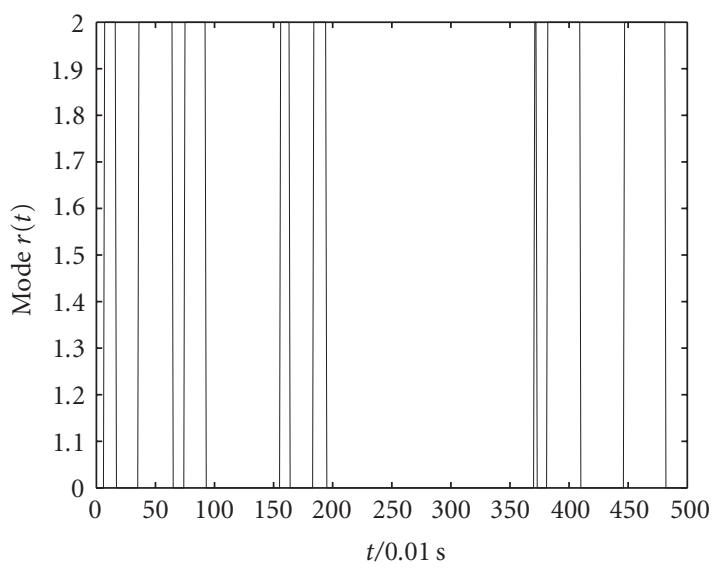

Figure 4.4. Regime transition in periodic-signal tracking.

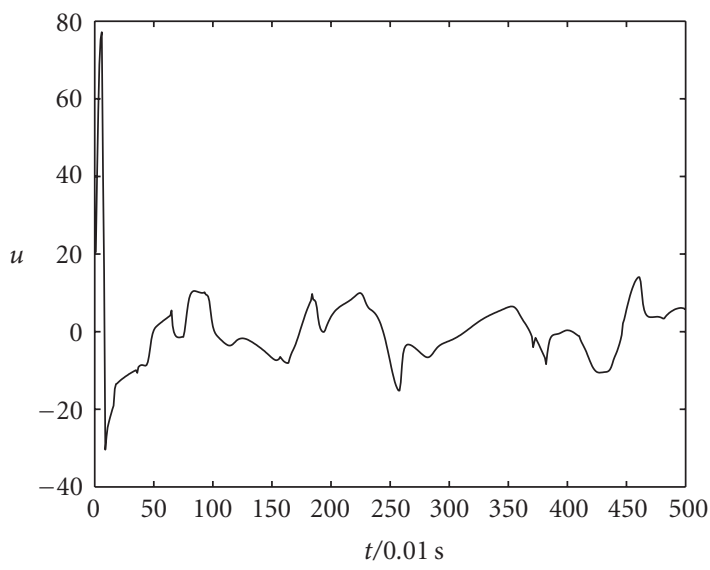

Figure 4.5. Control in periodic-signal tracking.

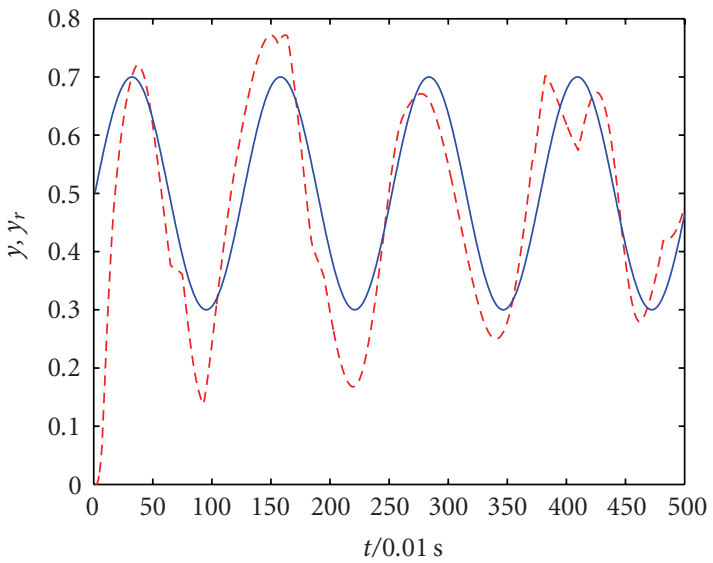

Figure 4.6. Output in periodic-signal tracking. 
The simulation results illustrate the global uniform ultimate boundedness of the closed-loop system.

\section{Conclusion}

The robust adaptive tracking problems of Markovian jump uncertain nonlinear parametric-strict-feedback systems with both parametric uncertainty and unknown nonlinearity are investigated. A robust adaptive control scheme was obtained by using a stochastic Lyapunov method and backstepping techniques, which guarantees that the closed-loop system is globally uniformly ultimately bounded. And the tracking error could be achieved to any prescribed accuracy. This work extends the class of Markovian jump nonlinear systems for which tracking problems are available and proposes a practical control design.

\section{Acknowledgment}

This work was supported by the Specialized Research Fund for the Doctoral Program of Higher Education of China under Grant 20050358044.

\section{References}

[1] M. D. S. Aliyu and E.-K. Boukas, $H_{\infty}$ control for Markovian jump nonlinear systems, 37th CDC, 1998, pp. 766-771.

[2] M. D. Fragoso, Discrete-time jump LQG problem, International Journal of Systems Science 20 (1989), no. 12, 2539-2545.

[3] Y. D. Ji and H. J. Chizeck, Jump linear quadratic Gaussian control in continuous time, IEEE Transactions on Automatic Control 37 (1992), no. 12, 1884-1892.

[4] M. S. Mahmoud, P. Shi, and A. Ismail, Robust Kalman filtering for discrete-time Markovian jump systems with parameter uncertainty, Journal of Computational and Applied Mathematics 169 (2004), no. 1, 53-69.

[5] X. Mao, Stability of stochastic differential equations with Markovian switching, Stochastic Processes and Their Applications 79 (1999), no. 1, 45-67.

[6] M. Mariton, Jump Linear Systems in Automatic Control, Marcel-Dekker, New York, 1990.

[7] B. Øksendal, Stochastic Differential Equations, Springer, New York, 2000.

[8] M. M. Polycarpou and P. A. Ioannou, A robust adaptive nonlinear control design, Automatica 32 (1996), no. 3, 423-427.

[9] S. Sathananthan and L. H. Keel, Optimal practical stabilization and controllability of systems with Markovian jumps, Nonlinear Analysis 54 (2003), no. 6, 1011-1027.

[10] P. Shi, E.-K. Boukas, and R. K. Agarwal, Kalman filtering for continuous-time uncertain systems with Markovian jumping parameters, IEEE Transactions on Automatic Control 44 (1999), no. 8, 1592-1597.

[11] C. Yuan and X. Mao, Asymptotic stability in distribution of stochastic differential equations with Markovian switching, Stochastic Processes and Their Applications 103 (2003), no. 2, 277-291. 


\section{Tracking problems of Markovian jump nonlinear systems}

[12] _ Robust stability and controllability of stochastic differential delay equations with Markovian switching, Automatica 40 (2004), no. 3, 343-354.

Jin Zhu: Department of Automation, University of Science and Technology of China (USTC), Hefei, Anhui 230027, China

E-mail address: zhujin@ustc.edu

Hong-Sheng Xi: Department of Automation, University of Science and Technology of China (USTC), Hefei, Anhui 230027, China

E-mail address: xihs@ustc.edu.cn

Hai-Bo Ji: Department of Automation, University of Science and Technology of China (USTC), Hefei, Anhui 230027, China

E-mail address: jihb@ustc.edu.cn

Bing Wang: Department of Automation, University of Science and Technology of China (USTC), Hefei, Anhui 230027, China

E-mail address: iceking@ustc.edu 


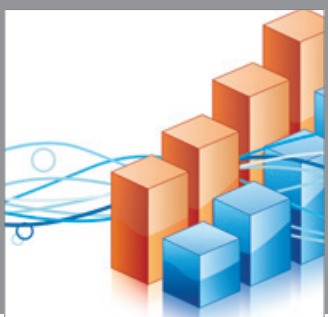

Advances in

Operations Research

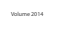

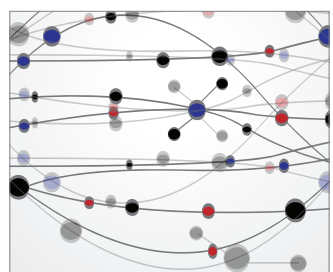

\section{The Scientific} World Journal
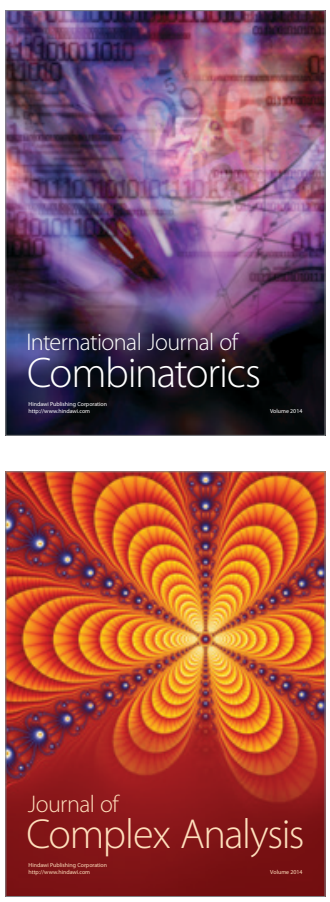

International Journal of

Mathematics and

Mathematical

Sciences
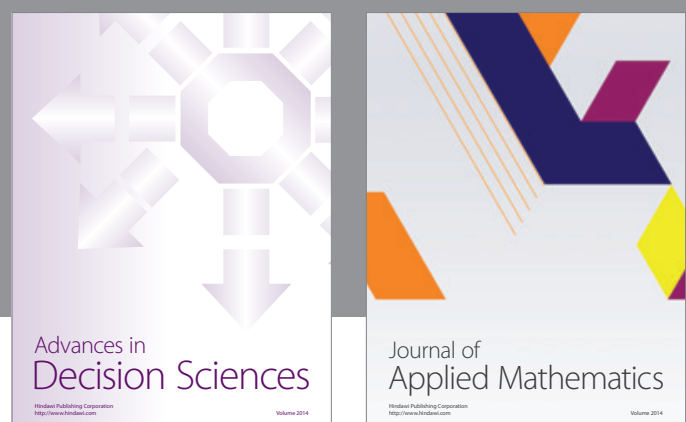

Journal of

Applied Mathematics
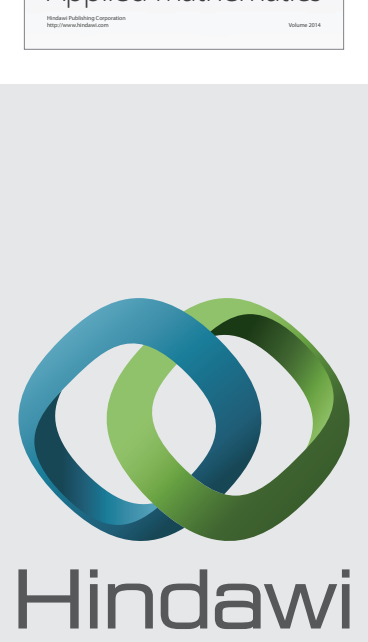

Submit your manuscripts at http://www.hindawi.com
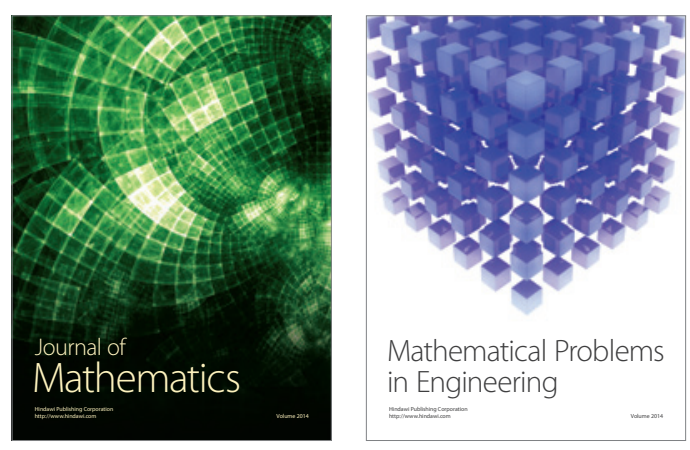

Mathematical Problems in Engineering
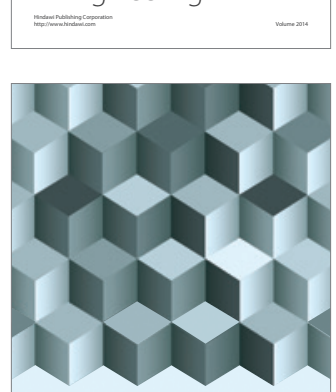

Journal of

Function Spaces
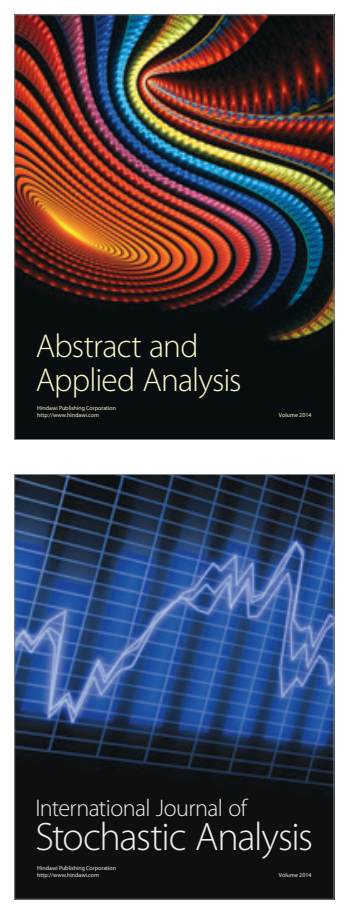

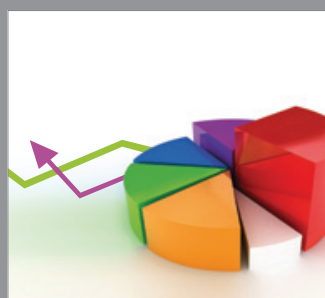

ournal of

Probability and Statistics

Promensencen
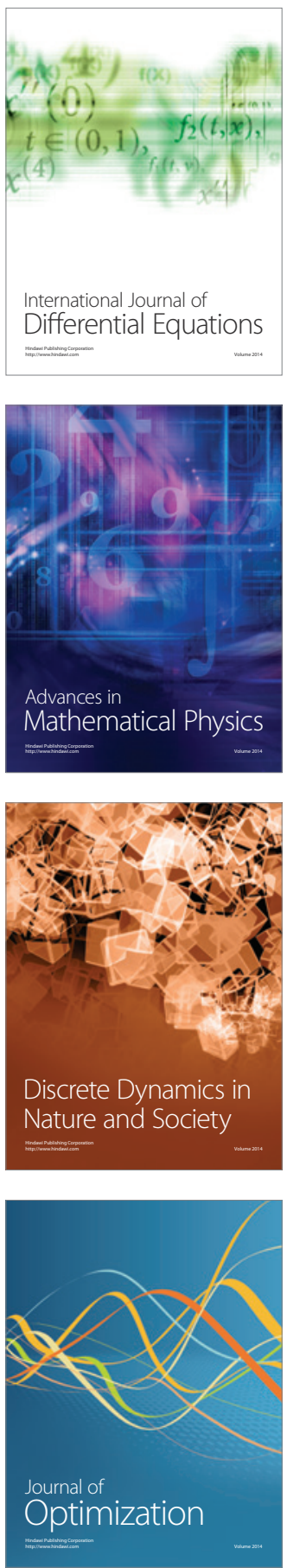